\title{
Macrosystems ecology: novel methods and new understanding of multi-scale patterns and processes
}

\author{
Songlin Fei · Qinfeng Guo · Kevin Potter
}

Received: 4 November 2015/Accepted: 14 November 2015/Published online: 24 November 2015

(C) Springer Science+Business Media Dordrecht 2015

\begin{abstract}
As the global biomes are increasingly threatened by human activities, understanding of macroscale patterns and processes is pressingly needed for effective management and policy making. Macrosystems ecology, which studies multiscale ecological patterns and processes, has gained growing interest in the research community. However, as a relatively new field in ecology, research in macrosystems ecology is facing various challenges. In this special issue, we highlight the following two latest exciting developments in this thriving field: (1) novel tools and methods and (2) new understandings on macroscale patterns and processes. While we believe that the contributions featured in this issue provide
\end{abstract}

Special issue: Macrosystems ecology: Novel methods and new understanding of multi-scale patterns and processes.

Guest Editors: S. Fei, Q. Guo, and K. Potter.

S. Fei $(\bowtie)$

Department of Forestry and Natural Resources, Purdue University, West Lafayette, IN, USA

e-mail: sfei@purdue.edu

Q. Guo

USDA Forest Service Southern Research Station,

Research Triangle Park, Durham, NC, USA

K. Potter

Department of Forestry and Environmental Resources, North Carolina State University, Research Triangle Park, Durham, NC, USA promising advancements in macrosystems ecology, we also see multiple challenges for future research including (1) multidisciplinary approaches for longterm and multiscale studies and (2) scaling local patterns and processes to broader scales.

Keywords Macrosystems ecology $\cdot$ Macroecology · Macroscale $\cdot$ Scaling $\cdot$ Heterogeneity

Macrosystems ecology, the study of ecological patterns and processes across multiple spatial and temporal scales, is a topic of growing interest. Macrosystems ecology has strong connections with landscape ecology, which also has deep emphases on spatial and temporal patterns, heterogeneity, and scale multiplicity (Forman 1995; Turner 2005; Wu 2013). Since the publication of the foundational paper by Brown and Maurer (1989), which coined the term of "macroecology", the field has experienced a rapid growth (Fig. 1). Macroecology is principally concerned with finding patterns in large-scale abundances and distributions and theorizing the reasons why these patterns exist (Brown 1995). It seeks generalized patterns at large spatial and temporal scales and statistical relationships that explain the distribution of biodiversity from a historical and geographical perspective (Brown 1995; Gaston and Blackburn 1999; Keith et al. 2012). 


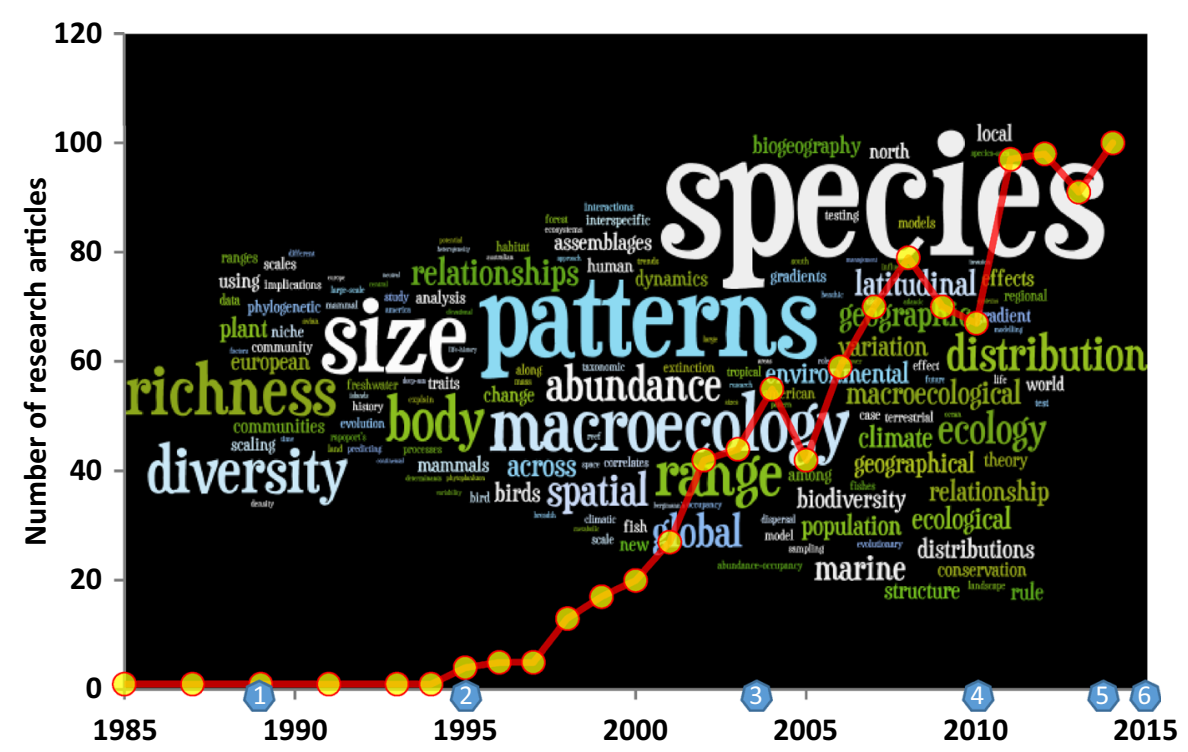

Fig. 1 The development and focus of macrosystems ecology based on articles in the ISI Web of Science database (webofknowledge.com) with keywords of macroecology, macrosystems biology, or macrosystems ecology. The red line indicates the number of articles published each year; the word cloud (produced in Wordle.net) indicates the frequency of words used in the article titles (larger font size is proportional to appearance frequency); the number at the bottom indicates

Macrosystems ecology expands upon macroecology by studying not only the large scale patterns, but also the interactions and feedbacks across scales. More importantly, it emphasizes the relationships among three critical multi-thematic components: (1) geophysical processes, (2) biological processes, and (3) socio-cultural processes (Heffernan et al. 2014). However, as highlighted by Beck et al. (2012) and mirrored by our word-cloud (Fig. 1), the predominant research in macrosystems ecology is still speciescentric and pattern-oriented. This pattern is likely due to the paucity of data, especially at regional and continental scales (Soranno and Schimel 2014), and to methodological challenges (Levy et al. 2014).

As the global biomes are increasingly threatened by invasive species, climate change, and land use change, understanding of macroscale patterns and processes is pressingly needed for effective management and policy making. In 2010, the U.S. National Science Foundation initiated a MacroSystems Biology program, which funded many macrosystems ecology projects (Gholz and Blood 2015), as well as inspired growing interest in the research community. Since important events in the development of the field: 1 Brown and Maurer (1989) paper in Science, 2 Brown (1995) macroecology book, 3 Blackburn and Gaston (2003) macroecology book, 4 initiation of the Macrosystems Biology program by U.S. National Science Foundation in 2010, 5 macrosystems ecology special issue edited by Soranno and Schimel (2014), and 6 this special issue

then, various novel methods have been developed and new understandings of macroscale patterns and processes have been gained. In this special issue, we highlight some of the latest developments in this thriving field.

\section{Novel tools and methods}

To enhance our understanding of how variations in fine-scale characteristics and interactions relate to broad-scale spatial and temporal patterns and processes, there is a clear need to obtain data from finegrained experimental and/or observational studies across a broad range of scales. For plant species, especially trees, many electronic databases of finescale occurrence/abundance data have been established, such as the National Forest Inventory database across multiple European countries and the Forest Inventory and Analysis database in the U.S. However, such fine-scale data are relatively sparse for animals.

To address data related challenges in macrosystem studies, (Bridge et al. 2015) offer an innovative 
approach using weather surveillance radar (WSR) to monitor (and to quantify) roosts of a wildlife species, the purple martin, at a regional scale. They also described specific features encountered at visited roost sites where exact coordinates were verified. Another approach is the use of citizen science, which has become a popular tool for macroecology studies in recent years. Work by McShea et al. (2015) is among the first that uses volunteers for the purpose of distributing camera traps and analyzing data submitted from those traps as an effective approach to wildlife monitoring at large scales. Their newly developed eMammal software program facilitates the utilization of citizen scientists in the streamlined collection and processing of a huge volume of photos and identification of mammals captured by remote cameras at the landscape to regional scales. Due to data availability, most studies on macroscale patterns are based on occurrence data, and not on abundance data. Bradley (2015) evaluated the ability of presence-only species distribution models to predict ranked abundance values. The study suggests that low-cost species occurrence data can be scaled up using climatic suitability niches to help describe macroscale patterns of abundance. Given the potential for using low-cost data to predict macroscale patterns of abundance, and the increased recent interest in abundance modelling, this work could have broader applications.

An important step toward the understanding of macroscale patterns and processes is the assessment, visualization and communication of the vast data involved in most macrosystems ecology studies. The representativeness and completeness (RAC) index developed by Fei and Yu (2015) provides a modelindependent assessment of data acceptability in species distribution models (SDMs), which are commonly used in macrosystems studies. The authors used a virtual species with different spatial clustering in a two dimensional environmental space (namely, precipitation and temperature). They found that RAC thresholds existed in determining the quality of occurrence data and recommended a RAC threshold value of 0.4 to ensure the accuracy of SDMs. The article provides great promise for designing effective field campaigns in capturing fine-scale data. Potter et al. (2015) developed a simple but useful tool for macroecological research and communication. Specifically, they presented a novel solution to a vexing problem in ecological monitoring by offering a scalable analytical framework to assess a variety of ecological metrics. Their approach allows effective display and analysis of fine-scale ecological data collected at large geographic scales to address macroscale ecological/management questions.

The topic of scale and heterogeneity is at the forefront of macrosystems ecology research, and there is a pressing need to develop methods that can model heterogeneity across scales. The study by Dixon Hamil et al. (2015) makes a novel contribution to macrosystems ecology by introducing a statistical technique in the form of mixed effect models for capturing heterogeneity across scales. Their proposed approach is advantageous because it is not always necessary to know the influential explanatory variables that cause spatial heterogeneity, and therefore the method requires no additional data to assess spatial heterogeneity. In addition, this approach can be applied to data having various distribution types and is easily executable using multiple statistical packages.

\section{Macroscale patterns and processes}

With the application of new methods and existing approaches in novel ways, several papers in this issue describe recent projects across a range of ecological fields, from species invasion and urban ecology to hydrology and climate change. These studies demonstrate the power and utility of macrosystem ecological approaches and tools to inform environmental policy and to improve our understanding of environmental dynamics across large areas.

These regions can span continents or even the entire globe. Riitters et al. (2015), for example, quantified the global net loss of forest area between 2000 and 2012. During that period, global forest area decreased 3.2 percent, but the amount of forest interior area declined by $9.9 \%$. These loss rates were consistent across most of the world, and demonstrate a widespread shift toward higher fragmentation of the world's remaining forests. Hall et al. (2015), meanwhile, applied finescale micrometeorogical data from representative sites across the United States in a novel exploration of continental-scale climatic patterns within common urban microenvironments. Specifically, they tested the urban homogenization hypothesis, which posits that ecological structure and function within urban 
ecosystems should exhibit high similarity compared to the native ecosystems they replaced. Their findings provide evidence that common residential land cover and structural characteristics do, in fact, lead to microclimatic convergence across diverse regions.

The key to understanding many ecological systems lies in illuminating the mechanisms that underlie observed patterns, which may operate at different scales than those at which the patterns are observed (Levin 1992). Elucidating the causes of ecological patterns and processes across broad areas, and within the context of spatial scale, requires innovative analytical methods, as noted above. In one such approach, Iannone et al. (2015) applied a mixedeffects modeling framework using data from 42,626 inventory plots across the eastern United States to assess whether native communities are resistant to biological invasions at macroscales and to determine the degree to which such biotic resistance relationships vary with scale or location. This novel modeling framework accounts for potential sub-regional and cross-scale variability in invader-native associations. The results indicate that native tree biomass and evolutionary diversity, but not species richness, are negatively associated with invader and establishment and thus are indicative of biotic resistance. Meanwhile, Rüegg et al. (2015) aimed to identify the spatial scales exhibiting and predicting heterogeneity in physical stream attributes based on data from five stream networks located in biomes spanning tropical forest to arctic tundra. They found that scaling an ecological process such as stream metabolism requires a framework that links habitat templates and ecological processes; such scaling should also consider within- and among-watershed variability to identify which properties of scaling functions may be applicable at the continental scale.

Predicting the extent and ecological impacts of global climate change is a promising area for the application of macrosystems ecology approaches and tools. Wang et al. (2015), for example, used a landscape modeling approach that incorporated three levels of spatial hierarchy to model climate-changerelated regional-scale shifts in forest composition across the Central Hardwood Forest Region of the United States. The authors suggest that it is essential to evaluate changes at multiple spatial and temporal scales when evaluating climate-associated changes in species composition.
An important need for climate change research is to better understand how the complexity of couplings and fluxes between the biosphere and the atmosphere impact the structure, function, and dynamics of terrestrial ecosystems (Baldocchi 2014; International Panel on Climate Change 2014). In this context, Ruddell et al. (2015) modeled the eco-climate macrostate, and its elasticity to seasonal air temperature and precipitation and to ecosystem phenophase, using time-series observations from seven eddy-covariance towers across the United States. The foundation of their analyses was a Dynamical Process Network approach, which uses information flow to model eco-climate system structure. They found that macrostate varies both by season and by ecosystem, with evergreen forests and grasslands highly elastic to seasonal air temperature and more likely than agricultural or deciduous systems to experience state changes with climate warming. Stark et al. (2015), meanwhile, propose that eco-climate "teleconnections," in which ecological changes in one area influence climate and associated ecological responses in another, should be considered when predicting the impacts of deforestation, afforestation and die-off associated with climate change, land conversion, and other phenomena. They illustrated potential ecoclimate teleconnections in a simulation that assumed complete tree cover loss in western North America, with results predicting subsequent drying and reduced net primary productivity in other areas of North America and beyond. They also introduce a framework for rapid field-based characterization of vegetation structure and energy balance for use in accurately modeling eco-climate teleconnections. As the climate warms, changes in the carbon balance of arctic tundra will play an important role in the global C balance, so Jiang et al. (2015) investigated the spatial distribution of soil organic matter and vegetation on the North Slope of Alaska and examined the effects of changes in nitrogen and phosphorus cycles on tundra $\mathrm{C}$ budgets under warming conditions. Their simulations showed substantial increases in $\mathrm{N}$ and $\mathrm{P}$ mineralization with climate warming, with consequent increases in nutrient availability to plants. This is expected to result in the net accumulation of $\mathrm{C}$ in tundra ecosystems, as $\mathrm{C}$ gains in vegetation would more than offset $\mathrm{C}$ losses from the soil. 


\section{Challenges and future directions}

Macrosystems ecology is still in an early stage of development and is facing many challenges such as paucity of region-wide fine-scale data, innovative modeling and analytical techniques, and novel frameworks that facilitate interdisciplinary solutions. Even with the advancement of technology such as high precision GIS and high spatial and spectral resolution remote sensing and with the implementation of region-wide networks such as the Long-Term Ecological Research Program (LTER) and the National Ecological Observation Network (NEON), there is still much room for development of novel approaches for data collection and analysis. This is especially needed for long-term temporal data at large scales. Therefore, multidisciplinary approaches are needed to incorporate long-term data or their surrogates into macrosystems ecology studies, which includes but not limit to: (1) phylogenetic information for evolutionary history, (2) paleo-data for geological and geographical history, (3) herbarium and museum specimens and long-term survey data for ecological history, and (4) historical demographic and land use and land change data for socioeconomic history.

A critical challenge in macrosystems ecology studies is the scaling issue. More specifically, we cannot simply examine local scale patterns and processes and linearly scale them up to broader scales due to inherent biases associated with spatial heterogeneity, cross-scale interactions, and non-linearity (Brown 1995; Peters et al. 2007). Fortunately, scaling has been a topic of interest in ecology for decades (Wiens 1989) and significant progress has been made about scaling in both natural and social sciences, particularly in landscape ecology (Wu 2007). Macrosystems ecology studies can benefit from borrowing existing methods such as those synthesized by $\mathrm{Wu}$ et al. (2006). With improved understanding of scaling and with recent computational and statistical advancements, we believe macrosystems ecology studies can be greatly advanced with the application of a cross-modeling framework that utilize an iterative exchange between (1) machine learning-based statistical models capable of capturing key within- and cross-scale interactions and tipping points among data challenged by different spatial and temporal resolutions and (2) agent-based computational experiments designed to capture uncertainties, path-dependence, and emergences that are common in complex systems.
Acknowledgments We thank Dr. Jianguo $\mathrm{Wu}$ for his assistance in making this special issue possible. Funding was provided through a National Science Foundation MacroSystems Biology grant (\#1241932) and through Cost Share Agreement 14-CS-11330110-042 between the USDA Forest Service and North Carolina State University.

\section{References}

Baldocchi D (2014) Measuring fluxes of trace gases and energy between ecosystems and the atmosphere: the state and future of the eddy covariance method. Glob Chang Biol 20(12):3600-3609

Beck J, Ballesteros-Mejia L, Buchmann CM et al (2012) What's on the horizon for macroecology? Ecography 35(8): 673-683

Blackburn TM, Gaston KJ (2003) Macroecology: concepts and consequences: 43rd symposium of the British Ecological Society. Cambridge University Press, Cambridge

Bradley B (2015) Predicting abundance with presence-only models. Landscape Ecol. doi:10.1007/s10980-015-0303-4

Bridge E, Pletschet S, Fagin T et al (2015) Persistence and habitat associations of Purple Martin roosts quantified via weather surveillance radar. Landscape Ecol. doi:10.1007/ s10980-015-0279-0

Brown JH (1995) Macroecology. University of Chicago Press, Chicago

Brown JH, Maurer BA (1989) Macroecology: the division of food and space among species on continents. Science 243(4895):1145-1150

Dixon Hamil K-A, Iannone Iii B, Huang W, Fei S, Zhang H (2015) Cross-scale contradictions in ecological relationships. Landscape Ecol. doi:10.1007/s10980-015-0288-Z

Fei S, Yu F (2015) Quality of presence data determines species distribution model performance: a novel index to evaluate data quality. Landscape Ecol. doi:10.1007/s10980-0150272-7

Forman RT (1995) Land mosaics: the ecology of landscapes and regions. Cambridge University Press, Cambridge

Gaston KJ, Blackburn TM (1999) A critique for macroecology. Oikos 84:353-368

Gholz H, Blood E (2015) MacroSystems Biology: stimulating new perspectives on scaling in ecology. Landscape Ecol. doi:10.1007/s10980-015-0300-7

Hall SJ, Learned J, Ruddell B et al (2015) Convergence of microclimate in residential landscapes 1 across diverse cities in the United States. Landscape Ecol. doi:10.1007/ s10980-015-0297-y

Heffernan JB, Soranno PA, Angilletta MJ et al (2014) Macrosystems ecology: understanding ecological patterns and processes at continental scales. Front Ecol Environ 12(1):5-14

Iannone BV, Potter KM, Dixon Hamil K et al (2015) Evidence of biotic resistance to invasions in forests of the Eastern USA. Landscape Ecol. doi:10.1007/s10980-015-0280-7

International Panel on Climate Change (2014) Climate change 2013: the physical science basis. Cambridge University Press, Cambridge 
Jiang Y, Rocha AV, Rastetter EB et al (2015) C-N-P interactions control climate driven changes in regional patterns of C storage on the North Slope of Alaska. Landscape Ecol. doi:10.1007/s10980-015-0266-5

Keith SA, Webb TJ, Böhning-Gaese K et al (2012) What is macroecology? Biol Lett. doi:10.1098/rsbl.2012.0672

Levin SA (1992) The problem of pattern and scale in ecology. Ecology 73(6):1943-1967

Levy O, Ball BA, Bond-Lamberty B et al (2014) Approaches to advance scientific understanding of macrosystems ecology. Front Ecol Environ 12(1):15-23

McShea W, Forrester T, Costello R, He Z, Kays R (2015) Volunteer-run cameras as distributed sensors for macrosystem mammal research. Landscape Ecol. doi:10. 1007/s10980-015-0262-9

Peters DC, Bestelmeyer B, Turner M (2007) Cross-scale interactions and changing pattern-process relationships: consequences for system dynamics. Ecosystems 10(5):790-796

Potter K, Koch F, Oswalt C, Iannone B III (2015) Data, data everywhere: detecting spatial patterns in finescale ecological information collected across a continent. Landscape Ecol. doi:10.1007/s10980-015-0295-0

Riitters KH, Wickham J, Costanza JK, Vogt P (2015) A global evaluation of forest interior area dynamics using tree cover data from 2000 to 2012. Landscape Ecol. doi:10.1007/ s10980-015-0270-9

Ruddell BL, Yu R, Kang M, Childers D (2015) Seasonally varied controls of climate and phenophase on terrestrial carbon dynamics: modeling eco-climate system state using dynamical process networks. Landscape Ecol. doi:10. 1007/s10980-015-0253-x
Rüegg J, Baker CL, Bowden WB et al (2015) Baseflow physical stream characteristics differ at multiple spatial scales in stream networks across diverse biomes. Landscape Ecol. doi:10.1007/s10980-015-0289-y

Soranno PA, Schimel DS (2014) Macrosystems ecology: big data, big ecology. Front Ecol Environ 12(1):3

Stark SC, Breshears DD, Garcia E et al (2015) Toward accounting for ecoclimate teleconnections: intra- and intercontinental consequences of altered energy balance after vegetation change. Landscape Ecol. doi:10.1007/s10980015-0282-5

Turner MG (2005) Landscape ecology: what is the state of the science? Annu Rev Ecol Evol Syst 36:319-344

Wang W, He H, Thompson F III, Fraser J, Dijak W (2015) Landscape- and regional-scale shifts in forest composition under climate change in the Central Hardwood Region of the United States. Landscape Ecol. doi:10.1007/s10980015-0294-1

Wiens JA (1989) Spatial scaling in ecology. Funct Ecol 3:385-397

Wu J (2007) Scale and scaling: a cross-disciplinary perspective. Key topics in landscape ecology. Cambridge University Press, Cambridge, pp 115-142

Wu J (2013) Key concepts and research topics in landscape ecology revisited: 30 years after the Allerton Park workshop. Landscape Ecol 28(1):1-11

Wu J, Jones B, Li H, Loucks OL (2006) Scaling and uncertainty analysis in ecology. Methods and applications. Springer, Dordrecht 